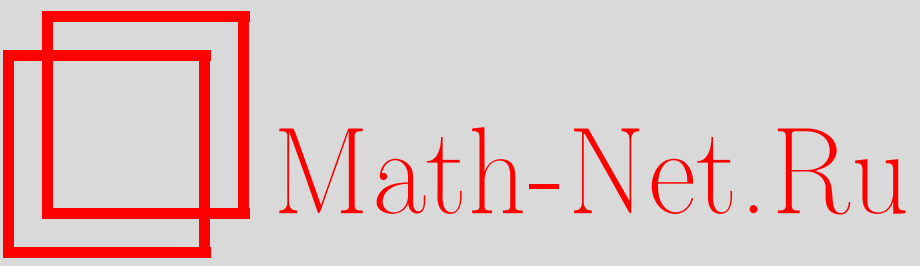

Ю. П. Вирченко, О. Л. Шпилинская, Точечные случайные поля с марковскими измельчениями и геометрия фрактально-неупорядоченных сред, $T M \Phi, 2000$, том 124, номер 3, 490-505

DOI: https://doi.org/10.4213/tmf653

Использование Общероссийского математического портала Math-Net.Ru подразумевает, что вы прочитали и согласны с пользовательским соглашением

http: //www . mathnet.ru/rus/agreement

Параметры загрузки :

IP : 52.90 .164 .192

26 апреля 2023 г., 17:46:18 
ТЕОРЕТИЧЕСКАЯ

И МАТЕМАТИЧЕСКАЯ

ФИЗИКА

Том 124, № 3

сентябрь, 2000

(C) 2000 г.

Ю. П. Вирченко*, О. Л. Шпилинская*

\section{ТОЧЕЧНЫЕ СЛУЧАЙНЫЕ ПОЛЯ С МАРКОВСКИМИ ИЗМЕЛЬЧЕНИЯМИ И ГЕОМЕТРИЯ ФРАКТАЛЬНО-НЕУПОРЯДОЧЕННЫХ СРЕД}

\footnotetext{
Дана общая конструкция вероятностной меры для описания стохастических фракталов, моделируюших фрактально-неупорядоченные среды. Для таких стохастических фракталов введено понятие метрически однородной фрактальной меры Хаусдорфа-Каратеодори, имеющей неслучайный тип. Выделен класс $\mathbf{F}[q]$ случайных точечных полей с марковскими измельчениями, для которых явным образом строится распределение вероятностей. Доказана теорема о том, что при некотором довольно слабом ограничении для случайных полей этого класса фрактальная размерность $D$ является самоусредняющейся величиной и с вероятностью 1 на таких фракталах определена фрактальная мера, обладающая неслучайным типом ( $D$-мера Хаусдорфа).
}

\section{1. ВВЕДЕНИЕ}

При исследовании методами статистической механики неупорядоченных систем, которые являются реалистическими моделями конденсированных сред, встречаюшихся в природе, приходится решать вопрос о вероятностном моделировании стохастической геометрии той структуры, на которой рассматривается изучаемое случайное поле (например, магнитное поле атомов среды) [1]. Для сред, неупорядоченность которых проистекает из метастабильности их термодинамического состояния с очень большим временем релаксации к истинному равновесию, указанное моделирование с необходимостью приходится осуществлять на основе феноменологических представлений, привлекая чисто статистические аргументы. При этом стохастическая геометрия указанных систем может быть весьма разнообразной. В простейшем случае неупорядоченность связана с нарушениями кристаллической решетки случайно расположенными с малой плотностью инородными примесями, центрами дилатации. Их геометрию в теории твердого тела описывают, как правило, пуассоновским точечным случайным полем с соответствуюшей плотностью. Противоположный в смысле используемых математических средств случай реализуется при описании гетерогенно-неупорядоченных структур. Используемые при этом точечные случайные поля являются сепарабельными [2].

${ }^{*}$ Институт монокристаллов НАН Украины, Харьков, Украина 
В частности, такие вероятностные модели описывают поликристаллические структуры [3]. Заметим, что здесь мы используем термин точечное случайное поле в более широком смысле, чем это обычно принято [4], обозначая им случайные точечные множества в пространстве $\mathbb{R}^{d}$.

В этой работе мы будем интересоваться промежуточным случаем как по математической конструкции, так и по физическому смыслу. А именно будем рассматривать среды, неупорядоченность которых невозможно охарактеризовать конкретными значениями одной или нескольких величин размерности длины, а, наоборот, их неупорядоченность характеризуется широким спектром масштабов, которые изменяются от межатомных расстояний до макроскопических размеров. О таких средах естественно говорить, что они являются фрактально-неупорядоченными.

Примерами фрактально-неупорядоченных сред, вероятностный формализм описания геометрии которых рассматривается в работе, являются такие, которые образуют сильно-пористые (губчатые) структуры. Мы будем рассматривать в некотором смысле идеализированные геометрические модели таких сред, когда пространственное расположение частиц образует в указанном вьше приближении эффективную геометрическую структуру с пренебрежимо малым объемом по сравнению с объемом пустот. В этом случае для задания геометрии неупорядоченной среды можно ввести такую метрическую характеристику, как фрактальная размерность, отличие которой от размерности физического пространства приводит, как известно (см., например, [5]), к появлению самоподобных функциональных зависимостей локальных физических характеристик среды от размеров областей, по которым производится усреднение.

Несмотря на то что математическая теория фракталов имеет давнюю историю и уже солидный стаж насчитывает практика применения фрактальных структур в теоретической физике, эта проблема остается малоизученной. Причины такого положения лежат в неординарной математической природе таких геометрических конструкций - точечных случайных полей на пространствах с дробной величиной фрактальной размерности. В настояшее время имеется несколько фундаментальных исследований, в которых, в частности, изучается задача построения распределений вероятностей для таких стохастических структур (см., например, $[6,7])$. В то же время эти исследования не ориентированы на физические приложения и поэтому их результаты не могут быть напрямую использованы для построения статистического описания фрактально-неупорядоченных сред. А именно в этих работах даже не ставится вопрос о конструкции таких стохастических фракталов, которые бы отвечали перечисляемым ниже требованиям.

При построении случайных точечных полей, описывающих фрактальные среды, необходимо позаботиться о том, чтобы их распределение вероятностей обладало определенными свойствами, наличие которых диктуется физическими соображениями. Так, вследствие пространственной однородности в среднем фрактально-неупорядоченных сред необходима также стохастическая пространственная однородность описывающих их случайных множеств точек. Необходима самоусредняемость фрактальной размерности этих случайных полей, т.к. эта величина феноменологически является 
достоверно измеряемым физическим параметром фрактально-неупорядоченной среды. Необходимо сушествование на стохастической фрактальной структуре такой меры, которая бы имела неслучайный mun (см. определение этого понятия в разделе 4). Наличие этого свойства позволяет вводить плотности распределения физических величин на стохастическом фрактале, т.е. вводить для каждой из них соответствующую локальную величину.

В данной работе мы строим класс конкретных моделей случайных точечных полей, которые называем полями с марковскими измельчениями. Среди таких геометрических структур имеется обширный класс стохастически пространственно-однородных. Для введенного класса моделей будет доказана теорема о самоусредняемости (неслучайности) их фрактальной размерности $D$ и для этой величины будет получена явная формула. Более того, будет доказано, что с вероятностью единица фрактальные меры на случайных реализациях являются $D$-мерами Хаусдорфа.

\section{2. КЛЕТОЧНОЕ ИЗМЕЛЬЧЕНИЕ ПРОСТРАНСТВА $\mathbb{R}^{d}$}

Разобьем все пространство $\mathbb{R}^{d}$ на непересекающиеся полуоткрытые справа кубы $\left\{A_{\mathbf{n}}^{(0)} ; \mathbf{n} \in \mathbb{Z}^{d}\right\}(\kappa л е т к и)$ с длиной ребра $L \in \mathbb{R}$, которые нумеруются целочисленными векторами $\mathbf{n} \in \mathbb{Z}^{d}$.

Исходя из разбиения $\left\{A_{\mathbf{n}}^{(0)}\right\}$ построим шаг за шагом последовательность

$$
\left\{\left\{A_{\mathbf{n}, \mathbf{n}_{1}, \ldots, \mathbf{n}_{m}}^{(m)}\right\} ; m=0,1,2, \ldots\right\}
$$

измельчающихся клеточных разбиений пространства $\mathbb{R}^{d}$, т.е. последовательность семейств

$$
\left\{A_{\mathbf{n}, \mathbf{n}_{1}, \ldots, \mathbf{n}_{m}}^{(m)} ; \mathbf{n} \in \mathbb{Z}^{d}, \mathbf{n}_{j} \in \mathfrak{I}_{N}^{d}, j=1,2, \ldots, m\right\}, \mathfrak{I}_{N}=\{0, \ldots, N-1\}
$$

непересекающихся клеток. Каждая клетка $A_{\mathbf{n}, \mathbf{n}_{1}, \ldots, \mathbf{n}_{m}}^{(m)}$ разбиения $m$-го порядка представляет собой полуоткрытый справа $d$-мерньй куб с длиной ребра $L / N^{m}$, где $N$ - натуральное число, большее 1 , которое будем назьвать степенью дробления. Ребра этих кубов ориентированы по некоторому ортогональному реперу $\{\mathbf{e}\}$ в $\mathbb{R}^{d}$. Полуоткрытость клеток понимается в том смысле, что по каждому направлению е кубы $A_{\mathbf{n}, \mathbf{n}_{1}, \ldots, \mathbf{n}_{m}}^{(m)}$ замкнуты слева и открыты справа. Таким образом, описание всех клеток разбиения порядка $m$ осушествляется упорядоченными наборами $\boldsymbol{\xi}=\left[\mathbf{n}, \mathbf{n}_{1}, \ldots, \mathbf{n}_{m}\right]$, которые будут в дальнейшем выполнять роль координат.

Последовательности разбиений, описанные в предыдущем разделе, будем называть клеточны.ми измельчениями пространства $\mathbb{R}^{d}$. Тогда числа $L, N$, репер $\{\mathbf{e}\}$ и нулевая точка разбиения $\left\{A_{\mathbf{n}}^{(0)}\right\}$ являются параметрами измельчения, которое мы кратко будем обозначать в виде $\left\{A_{\boldsymbol{\xi}}^{(m)} ; m \in \mathbb{N}\right\}$.

Описание любого множества $X$ точек в $\mathbb{R}^{d}$ осушествляется указанием для любого $m=0,1,2, \ldots$ множества тех клеток $A_{\boldsymbol{\xi}}^{(m)}$ из разбиения порядка $m$, для которых 
$X \cap A_{\boldsymbol{\xi}}^{(m)} \neq \varnothing$. При этом объединение всех таких клеток при фиксированном $m$ образует множество $X^{(m)}$, которое мы будем назьвать клеточным покрытием $m$-го порядка. Тогда члены последовательности $\left\{X^{(m)} ; m=0,1,2, \ldots\right\}$ удовлетворяют естественному условию $X^{(m+1)} \subset X^{(m)}$, следствием которого является существование теоретико-множественного предела

$$
X=\lim _{m \rightarrow \infty} X^{(m)}
$$

Поскольку в этой работе нашей задачей является доказательство теорем о самоусредняемости фрактальной размерности и о неслучайности типа фрактальной меры безотносительно к размеру области, в которой изучается стохастическая геометрическая структура, то достаточно ограничиться пространством погружения для конструируемого стохастического фрактала в виде куба $[0, L)^{d}=\Lambda$. В связи с этим первую метку $\mathbf{n}$ в индексе, нумеруюшем клетки разбиений, можно исключить из рассмотрения. Поэтому считаем далее, что $\boldsymbol{\xi}=\left[\mathbf{n}_{1}, \ldots, \mathbf{n}_{m}\right]$ - вектор длины $m$, описывающий точку в кубе $\Lambda$. Каждая компонента этого вектора является $m$-значной $N$-ичной дробью. Между всеми такими точками и всеми клетками из разбиения порядка $m$ существует взаимно однозначное соответствие. Множество всех возможных векторов $\left[\mathbf{n}_{1}, \ldots, \mathbf{n}_{m}\right]$, связанных с разбиением порядка $m$, будем обозначать символом $\mathfrak{K}_{m}$.

Рассмотрим произвольную точку $\mathbf{x} \in \Lambda$. Пусть эта точка принадлежит клетке $A_{\boldsymbol{\xi}}^{(l)}$, $\boldsymbol{\xi} \in \mathfrak{K}_{l}$, из разбиения $l$-го порядка. Тогда метку $\boldsymbol{\xi}$ этой клетки назовем проекцией точки $\mathbf{x}$ на разбиение $\left\{A_{\boldsymbol{\xi}}^{(l)} ; \boldsymbol{\xi} \in \mathfrak{K}_{l}\right\}$. Операцию проектирования будем обозначать следующим образом: $\boldsymbol{\xi} \equiv \mathbf{K}_{l}(\mathbf{x})$.

Введя понятие проекции точки на $\left\{A_{\boldsymbol{\xi}}^{(l)} ; \boldsymbol{\xi} \in \mathfrak{K}_{l}\right\}$, определим теперь естественным образом проекцию $\mathbf{K}_{l}(X)$ произвольного множества точек $X \subset \Lambda$ на это разбиение,

$$
\mathbf{K}_{l}(X)=\left\{\boldsymbol{\xi}: \boldsymbol{\xi}=\mathbf{K}_{l}(\mathbf{x}), \mathbf{x} \in X\right\}=\left\{\boldsymbol{\xi} \in \mathfrak{K}_{l}: X \cap A_{\boldsymbol{\xi}}^{(l)} \neq \varnothing\right\}
$$

В частности, если множество $H \subset \mathfrak{K}_{m}$ при $l<m$, то его проекция на $\mathfrak{K}_{l}$ состоит из векторов

$$
\mathbf{K}_{l}(H)=\left\{\left[\mathbf{n}_{1}, \ldots, \mathbf{n}_{l}\right] ;\left[\mathbf{n}_{1}, \ldots, \mathbf{n}_{l}, \ldots, \mathbf{n}_{m}\right] \in H\right\} .
$$

В этих обозначениях $\mathfrak{K}_{m}=\mathbf{K}_{m}(\Lambda)$ и клеточное покрытие $X^{(m)}$ множества $X$ описывается формулой

$$
X^{(m)}=\bigcup_{\boldsymbol{\xi} \in \mathbf{K}_{m}(X)} A_{\boldsymbol{\xi}}^{(m)} .
$$

\section{3. ФРАКТАЛЬНАЯ РАЗМЕРНОСТЬ И СТОХАСТИЧЕСКИЕ МЕРЫ}

Рассмотрим произвольное клеточное разбиение $\left\{A_{\boldsymbol{\xi}}^{(m)} ; \boldsymbol{\xi} \in \mathfrak{K}_{m}\right\}$ порядка $m$ пространства погружения $\Lambda$. Его кубические клетки имеют линейный размер $h=L / N^{m}$. Пусть $X$ - произвольное множество точек из $\Lambda$ и $X^{(m)}$ - его покрытие клетками разбиения $m$-го порядка. Это покрытие представляет собой наименьший из многогранников, составленных из клеток разбиения $\left\{A_{\boldsymbol{\xi}}^{(m)}\right\}$, содержащих точки из $X$. 
Пусть $|G| \equiv \operatorname{Card}(G)$ - число клеток в конечном множестве $G$. Введем величину $D$, определяемую формулой

$$
D=\sup \left\{\alpha: \lim _{h \rightarrow 0} h^{\alpha}\left|X^{(m)}\right|=0\right\}
$$

Эта величина в теории фракталов обычно именуется клеточной размерностью. В этой работе мы будем называть ее фрактальной размерностью, в отличие от общепринятой терминологии. Оправданием такой замены может служить то, что именно величина (2) определяется экспериментально при исследовании физических фракталов.

Физические величины на структурах описываются аддитивными функционалами (мерами) от компактных пространственных областей $G \subset \Lambda$ - областей измерения, в которых расположена изучаемая структура. Они описьвают суммарное значение измеряемой физической величины, носителями которой являются точки структуры, вырезаемые каждой из этих областей $G$.

Меры на фрактальных структурах, описывающие распределение физических величин, связанных с точками фрактальной структуры, которая имеет фрактальную размерность, отличную от размерности пространства погружения, должны вводиться способом, отличным от обычно применяемого для "нормальных" геометрических объектов. Известно, что для введения мер на фрактальных геометрических объектах подходят так называемые меры Хаусдорфа-Каратеодори [5], которые мы далее называем фрактальньцми мерами. Они непосредственно связаны с величиной фрактальной размерности изучаемой структуры. Дадим их определение.

Пусть $X$ - точечное множество в $\Lambda$ и $s(x)$ - фиксированная положительная возрастаюшая функция на $[0, \infty)$. Обозначим символом $\mu(X \cap G ; s)$ фрактальную меру, соответствуюшую функции $s(x)$, подмножества $X \cap G$, вырезаемого пространственной областью $G$. Эта величина определяется формулой

$$
\mu(X \cap G ; s)=\lim _{h \rightarrow 0} \sum_{\boldsymbol{\xi}} s\left(\operatorname{diam} A_{\boldsymbol{\xi}}^{(m)}\right),
$$

где суммирование осуществляется по покрытию клетками $A_{\boldsymbol{\xi}}^{(m)}$. Если положить в (3) $s(x)=x^{\alpha}$, то $\mu(\cdot ; s)$ преврашается в так называемую $\alpha$-меру Хаусдорфа. Для этой меры мы будем использовать обозначение $\mu_{\alpha}(\cdot)$. На данное нами определение меры $\mu(\cdot ; s)$ и на ее частный случай меры $\mu_{\alpha}(\cdot)$ распространяется оговорка, сделанная нами при определении фрактальной размерности.

Формула (3) действительно задает меру, если имеюшаяся в ней операция предельного перехода не приводит к тривиальному результату, т.е. не принимает сразу для всех $G$ значения 0 (или $\infty$ ). Очевидно, что для этого необходимо, чтобы фрактальная размерность $D$ множества $X \cap G$ была равна $\alpha$. Однако это условие не является достаточным. Но в любом случае можно утверждать, что фрактальная мера $\mu(\cdot ; s)$ конечна тогда и только тогда, когда существует медленно изменяющаяся в нуле функция $f(x)$, т.е. $\lim _{x \rightarrow 0} f(x+z) / f(x)=1$ при любом $z>0$, такая, что $s(x)=x^{D} f(x)$. Функция 
$f(x)$ определена при этом с точностью до произвольного множителя $g(x)$ такого, что $g(0) \neq 0, \infty$.

Нас будут далее интересовать только конечные меры $\mu(\cdot ; s)$, для которых $\mu(X \cap$ $G ; s)<\infty$ для всех $G \subset \Lambda$.

Множество $X$ назовем метрически однородным относительно меры $\mu(\cdot ; s)$, если эта мера конечна и для любой области $G$, содержашей точки множества $X$, выполняется условие $\mu(X \cap G ; s) \neq 0$. В этом случае можно говорить о едином для всего множества $X$ типе меры, определяемом фиксированной функцией $s(x)$.

Будем теперь вместо фиксированного точечного множества $X$ рассматривать случайные реализации $\mathfrak{X}$ стохастического фрактала $\{\mathfrak{X}\}$. Данные выше определения, разумеется, применимы и для каждой такой фиксированной реализации. Но при этом фрактальная размерность и, следовательно, фрактальные меры являются функционалами от $\mathfrak{X}$. Поэтому в общем случае эти величины должны пониматься как случайные. Такое положение неудовлетворительно с физической точки зрения. Так, при случайности величины фрактальной размерности не имеет смысла говорить об ее использовании в качестве характеристического параметра для фрактальных структур статистической физики. В то же время из практики известно, что фрактальная размерность является достаточно хорошей экспериментальной характеристикой. Это наводит на мысль, что в макроскопических системах статистической физики фрактальная размерность эффективно не должна быть случайной или, как принято выражаться в статистической физике, является самоусредняющейся величиной. Математическая проблема, возникаюшая в связи с этим, - это нахождение критерия, устанавливаюшего самоусредняемость фрактальной размерности для достаточно широкого класса реалистических моделей стохастических фракталов.

Фрактальная мера $\mu(\mathfrak{X} \cap G ; s)$, вводимая на стохастическом фрактале, является функционалом от $\mathfrak{X}$, принимающим, вообще говоря, случайные значения. При этом случайные реализации $\mathfrak{X}$ определяют выбор функции $s(\cdot)$ с точностью до несушественного множителя. Тогда эта функция в обшем случае также является случайной и, более того, может зависеть от $G$, т.е. мера $\mu(\cdot ; s)$ может не быть метрически однородной с вероятностью 1. Конечно же, такое положение неудовлетворительно при моделировании фрактально-неупорядоченных сред. В связи с этим мы далее интересуемся фрактальными мерами, являющимися метрически однородными с вероятностью 1 , и выделяем среди этого класса мер фрактальные меры неслучайного типа. Таковыми мы будем называть меры $\mu(\cdot ; s)$, у которых функция $s(\cdot)$ может быть сделана неслучайной.

Заметим, что стохастические фракталы $\{\mathfrak{X}\}$, которые обладают неслучайной фрактальной размерностью $D$, не зависящей от области $G$, и, с вероятностью $1, D$-мерой, автоматически являются с той же вероятностью метрически однородными и вводимая на них фрактальная мера имеет неслучайньй тип $s(x)=x^{D}$.

\section{4. ВЕРОЯТНОСТНОЕ ОПИСАНИЕ СТОХАСТИЧЕСКИХ ФРАКТАЛОВ}

Обсудим проблему построения распределений вероятностей для случайных множеств 
$\{\mathfrak{X}\}$ в компактном пространстве погружения $\Lambda$ на основе его клеточных измельчений $\left\{A_{\boldsymbol{\xi}}^{(m)} ; m \in \mathbb{N}\right\}$.

Так как согласно (1) для описания любого множества $X$ в $\Lambda$ достаточно определить его проекции $X^{(m)}$ при всех значениях $m=1,2,3, \ldots$, то для вероятностного описания любого случайного множества $\{\mathfrak{X}\}$ достаточно указать последовательность распределений вероятностей $\left\{P_{m}(\cdot) ; m=1,2, \ldots\right\}$ для случайных реализаций проекций $\mathfrak{X}^{(m)}$. Эти распределения определяются формулой

$$
P_{m}(H)=\mathbf{P}\left\{\mathfrak{X}: \mathbf{K}_{m}(\mathfrak{X})=H\right\}, \quad m=1,2, \ldots .
$$

Каждое из этих распределений заведомо существует, т.к. любая алгебра $\mathbb{S}_{m}\left(\left\{A^{(l)}\right\}\right)$ случайных событий, порожденная попарно несовместимыми событиями $\mathfrak{D}_{m}(H) \equiv\{\mathfrak{X}$ : $\left.\mathbf{K}_{m}(\mathfrak{X})=H\right\}$ со всевозможными $H \subset \mathfrak{K}_{m}$, состоит из конечного набора элементов при любом $m$.

Функции $P_{m}(\cdot)$ должны удовлетворять условиям нормированности

$$
\sum_{H \subset \mathfrak{K}_{m}} P_{m}(H)=1, \quad m=1,2, \ldots
$$

Кроме того, функции распределения $P_{m}(\cdot)$ различных порядков $m$ должны вследствие подчинения $\mathfrak{X}^{(m+1)} \subset \mathfrak{X}^{(m)}$ удовлетворять условиям согласованности, для формулировки которых нам потребуются следующие определения.

Пусть $H \subset \mathfrak{K}_{m}$. Тогда для каждого $l<m$ множество $H$ представимо в виде объединения непересекающихся классов $\left\{\mathbf{S}_{l}(\boldsymbol{\xi}, H) ; \boldsymbol{\xi} \in \mathbf{K}_{l}(H)\right\}$,

$$
H=\bigcup_{\boldsymbol{\xi} \in \mathbf{K}_{l}(H)} \mathbf{S}_{l}(\boldsymbol{\xi}, H),
$$

где класс, отвечающий точке $\boldsymbol{\xi} \in \mathbf{K}_{l}(H)$, определяется формулой

$$
\mathbf{S}_{l}(\boldsymbol{\xi}, H)=\left\{\boldsymbol{\eta} \in H: \mathbf{K}_{l}(\boldsymbol{\eta})=\boldsymbol{\xi}\right\}
$$

Определим операцию $\mathbf{T}_{l}(\cdot ; H)$ для $H \in \mathfrak{K}_{m}, m>l$, которая сопоставляет каждому классу $\mathbf{S}_{l}(\boldsymbol{\xi}, H)$, помеченному точкой $\boldsymbol{\xi} \in \mathbf{K}_{l}(H)$, подмножество целочисленных $d$-мерных векторов из набора $\Im_{N}^{d}$. Это подмножество состоит из $(l+1)$-х компонент векторов, входящих в класс $\mathbf{S}_{l}(\boldsymbol{\xi}, H)$,

$$
\mathbf{T}_{l}(\boldsymbol{\xi}, H)=\left\{\mathbf{n}_{l+1}:\left[\boldsymbol{\xi}, \mathbf{n}_{l+1}, \ldots, \mathbf{n}_{m}\right] \in \mathbf{S}_{l}(\boldsymbol{\xi}, H)\right\}
$$

Пусть $G \subset \mathfrak{K}_{m+1}$. Обозначим $H=\mathbf{K}_{m}(G)$ и пусть $\boldsymbol{\xi} \in H$. Разобьем множество $G$ на классы $\mathbf{S}_{m}(\boldsymbol{\xi}, G)$, которые не пусты в том и только в том случае, если $\boldsymbol{\xi} \in H$. Тогда имеет место соотношение

$$
\sum_{G \subset \mathfrak{K}_{m+1}} \cdots=\sum_{H \subset \mathfrak{K}_{m}} \sum_{\left\{\mathbf{S}_{m}(\boldsymbol{\xi}, G) ; \boldsymbol{\xi} \in H\right\}} \cdots
$$


где знак суммы $\sum_{\left\{\mathbf{S}_{m}(\boldsymbol{\xi}, G) ; \boldsymbol{\xi} \in H\right\}}$ обозначает последовательное суммирование по точкам из всех возможных классов эквивалентности, которые рассматриваются как функция от $\boldsymbol{\xi} \in H \subset \mathfrak{K}_{m}$.

Условия согласованности для набора распределений $\left\{P_{m}(\cdot)\right\}$ имеют вид

$$
\sum_{\left\{\mathbf{S}_{m}(\boldsymbol{\xi}, G) ; \boldsymbol{\xi} \in \mathbf{K}_{m}(G)\right\}} P_{m+1}(G)=P_{m}\left(\mathbf{K}_{m}(G)\right) .
$$

Они с необходимостью следуют из соотношения $\mathbf{K}_{m}\left(\mathfrak{X}^{(m+1)}\right)=\mathfrak{X}^{(m)}$ и определения (4) функций $P_{m}(\cdot)$. Очевидным образом равенство $(7)$ является также и достаточным для того, чтобы функция распределения $P_{m}(\cdot)$ давала бы те значения вероятностей для событий $\mathfrak{D}_{m}(H)$, которые они имеют, если рассматривать $\mathfrak{D}_{m}(H)$ как элементы алгебры $\mathbb{S}_{m}\left(\left\{A^{(l)}\right\}\right)$. По этой причине, в частности, соотношения $(7)$ не находятся в противоречии с условием нормированности (5).

Рассмотрим теперь минимальную $\sigma$-алгебру, содержашую все алгебры $\mathbb{S}_{m}\left(\left\{A_{\boldsymbol{\xi}}^{(l)}\right\}\right)$. Эту $\sigma$-алгебру будем обозначать символом $\mathbb{S}_{\infty}\left(\left\{A_{\boldsymbol{\xi}}^{(l)}\right\}\right)$. Очевидно, что $\mathbb{S}_{m+1}\left(\left\{A_{\boldsymbol{\xi}}^{(l)}\right\}\right) \supset$ $\mathbb{S}_{m}\left(\left\{A_{\boldsymbol{\xi}}^{(l)}\right\}\right)$, т.е. последовательность $\left\{\mathbb{S}_{m}\left(\left\{A_{\boldsymbol{\xi}}^{(l)}\right\}\right) ; m=1,2, \ldots\right\}$ образует монотонный класс (см., например, [8]) и

$$
\mathbb{S}_{\infty}\left(\left\{A_{\boldsymbol{\xi}}^{(l)}\right\}\right)=\lim _{m \rightarrow \infty} \mathbb{S}_{m}\left(\left\{A_{\boldsymbol{\xi}}^{(l)}\right\}\right)
$$

Поэтому вероятность любого события $\mathfrak{D}$ из $\mathbb{S}_{\infty}\left(\left\{A_{\boldsymbol{\xi}}^{(l)}\right\}\right)$ может быть определена по формуле

$$
\mathbf{P}\{\mathfrak{D}\}=\lim _{m \rightarrow \infty} \mathbf{P}\left\{\mathbf{K}_{m}(\mathfrak{D})\right\},
$$

т.е. она полностью определяется последовательностью функций $\left\{P_{m}(\cdot) ; m=1,2, \ldots\right\}$. Учитывая сделанное выше замечание о согласованности функций $P_{m}(\cdot)$ различных порядков, сформулируем в виде отдельной теоремы полученные выводы.

ТеОрема 1. Каждый набор неотрицательных функиий $\left\{P_{m}(\cdot) ; m=1,2, \ldots\right\}$, определенных соответственно на множсествах из $\mathbb{S}_{m}\left(\left\{A_{\boldsymbol{\xi}}^{(l)}\right\}\right)$ и удовлетворяющих условиям согласованности (7) и нормированности (5), однозначно задает распределение вероятностей на б-алгебре $\mathbb{S}_{\infty}\left(\left\{A_{\boldsymbol{\xi}}^{(l)}\right\}\right)$.

\section{5. ТОЧЕЧНЫЕ СЛУЧАЙНЫЕ ПОЛЯ С МАРКОВСКИМИ ИЗМЕЛЬЧЕНИЯМИ}

В этом разделе мы построим распределения вероятностей посредством конструкции, описанной в теореме 1 .

Члены последовательности $\left\{\left\{P_{m}(H) ; H \subset \mathfrak{K}_{m}\right\} ; m=1,2, \ldots\right\}$, определяющей распределение вероятностей на $\mathbb{S}_{\infty}\left(\left\{A_{\boldsymbol{\xi}}^{(l)}\right\}\right)$, связаны соотношением

$$
P_{m+1}(H)=Q_{m}\left(H \mid \mathbf{K}_{m}(H)\right) P_{m}\left(\mathbf{K}_{m}(H)\right),
$$

6 Теоретическая и математическая физика, т. 124, № 3, 2000 г. 
где $Q_{m}\left(H \mid H^{\prime}\right)$ - условная вероятность:

$$
Q_{m}\left(H \mid H^{\prime}\right)=\mathbf{P}\left\{\mathfrak{X}: \mathbf{K}_{m+1}(\mathfrak{X})=H \mid \mathbf{K}_{m}(\mathfrak{X})=H^{\prime}\right\} .
$$

Формула (8) представляет собой общее решение соотношений согласованности для последовательности распределений $\left\{P_{m}(H) ; m=1,2, \ldots\right\}$, где $Q_{m}\left(H \mid \mathbf{K}_{m}(H)\right)$ - произвольная функция при $H \subset \mathfrak{K}_{m+1}$, являюшаяся условной вероятностью. Она определяется на последовательностях $\left\{G_{m} ; m=1,2, \ldots\right\}$ множеств, обладающих свойствами $G_{m} \subset \mathfrak{K}_{m}$ и $\mathbf{K}_{m}\left(G_{m+1}\right)=G_{m}$. Определим для каждого $\boldsymbol{\xi} \in H$ множества $\mathbf{T}_{m}(\boldsymbol{\xi} ; G)=$ $\left\{\mathbf{n} \in \mathfrak{I}_{N}^{d}:(\boldsymbol{\xi}, \mathbf{n}) \in G\right\} \neq \varnothing$ и дадим следуюшее определение математического объекта, который является предметом изучения в этой работе.

Точечное случайное поле в $\Lambda$ будем называть полем с марковским измельчением, если условная вероятность $Q_{m}\left(H \mid H^{\prime}\right)(9)$ представима формулой

$$
Q_{m}\left(H \mid \mathbf{K}_{m}(H)\right)=\prod_{\boldsymbol{\xi} \in \mathbf{K}_{m}(H)} q\left(\mathbf{T}_{m}(\boldsymbol{\xi} ; H)\right),
$$

где $q(\cdot) \geqslant 0$ и виполняется условие

$$
\sum_{\varnothing \neq \sigma \subset \Im_{N}^{d}} q(\sigma)=1
$$

Очевидно, что совокупность величин $\left\{q(\sigma) ; \sigma \subset \mathfrak{I}_{N}^{d}\right\}$, которая дополнена элементом $q(\varnothing)=0$, образует распределение вероятностей на $\mathfrak{L}_{N}=2^{\mathfrak{J}_{N}^{d}}$, причем условие (11) обеспечивает сушествование с вероятностью 1 непустого множества $\mathfrak{X}$, покрываемого всеми множествами $H=\mathbf{K}_{m}(\mathfrak{X})$. Таким образом, введенные случайные множества полностью характеризуются функцией $q(\cdot)$ на $\mathfrak{L}_{N}$, и поэтому мы будем обозначать класс этих точечных случайных полей символом $\mathbf{F}[q]$. При конкретном выборе функции $q(\cdot)$ они изучались в работе [9]. Можно проследить связь этих случайных полей с фрактальными функциями, вводимыми в книге [6].

Покажем, что данное выше определение корректно, т.е. величина $Q_{m}\left(H \mid H^{\prime}\right)$, заданная формулой (10), действительно является условной вероятностью. Для этого необходимо убедиться в справедливости тождества

$$
\sum_{\left\{H \subset \mathfrak{K}_{m+1} ; \mathbf{K}_{m}(H)=H^{\prime}\right\}} Q_{m}\left(H \mid H^{\prime}\right)=1 .
$$

Применяя (6) с переменой местами множеств $G=\mathbf{K}_{m}(H)$ и $H$, получим

$$
\begin{gathered}
\sum_{\left\{H \subset \mathfrak{K}_{m+1}: \mathbf{K}_{m}(H)=H^{\prime}\right\}} Q_{m}\left(H \mid H^{\prime}\right)=\sum_{G \subset \mathfrak{K}_{m}} \sum_{\left\{\mathbf{S}_{m}(\boldsymbol{\xi}, H) ; \boldsymbol{\xi} \in G\right\}} Q_{m}\left(H \mid \mathbf{K}_{m}(H)\right)= \\
=\sum_{\left\{\mathbf{T}_{m}(\boldsymbol{\xi}, H) ; \boldsymbol{\xi} \in H^{\prime}\right\}} \prod_{\boldsymbol{\xi} \in H^{\prime}} q\left(\mathbf{T}_{m}(\boldsymbol{\xi}, H)\right)= \\
=\sum_{\{\sigma(\boldsymbol{\xi}) ; \boldsymbol{\xi} \in G\}} \prod_{\boldsymbol{\xi} \in G} q(\sigma(\boldsymbol{\xi}))=\prod_{\boldsymbol{\xi} \in G}\left(\sum_{\sigma \subset \Im_{N}^{d}} q(\sigma)\right)=1,
\end{gathered}
$$


где учтено, что в первой сумме только одно слагаемое с $H^{\prime}=\mathbf{K}_{m}(H)$ не равно нулю.

Точно так же показывается, что для распределений $P_{m}(\cdot)$ введенных случайных множеств вьполняются условия согласованности (7). Используя формулы определений (8), (10) и (11), получим

$$
\begin{aligned}
& \quad \sum_{\left\{\mathbf{S}_{m}(\boldsymbol{\xi}, H) ; \boldsymbol{\xi} \in \mathbf{K}_{m}(H)\right\}} P_{m+1}(H)= \\
& =\sum_{\left\{\sigma(\boldsymbol{\xi}) \subset \mathbf{T}_{m}(\boldsymbol{\xi}, H) ; \boldsymbol{\xi} \in \mathbf{K}_{m}(H)\right\}} P_{m}\left(\mathbf{K}_{m}(H)\right) \prod_{\boldsymbol{\xi} \in \mathbf{K}_{m}(H)} q(\sigma(\boldsymbol{\xi}))= \\
& =P_{m}\left(\mathbf{K}_{m}(H)\right) \prod_{\boldsymbol{\xi} \in \mathbf{K}_{m}(H)}\left(\sum_{\sigma(\boldsymbol{\xi}) \subset \mathbf{T}_{m}(\boldsymbol{\xi}, H)} q(\sigma(\boldsymbol{\xi}))\right)=P_{m}\left(\mathbf{K}_{m}(H)\right) .
\end{aligned}
$$

Заметим, что класс случайных множеств с марковским измельчением содержит подкласс точечных случайных полей, который может играть особую роль в статистической механике фрактально-неупорядоченных структур. Этот подкласс характеризуется свойством $q(\sigma)=q_{|\sigma|}$, где набор чисел $q_{l}>0, l=1, \ldots, 2^{N^{d}}$, образует распределение вероятностей. Легко показать, что случайные множества указанного типа являются стохастически пространственно-однородными.

\section{6. МЕТОД ПРОИЗВОДЯЩЕЙ ФУНКЦИИ}

Исследование точечных случайных полей класса $\mathbf{F}[q]$ основано на том, что последовательность $\left\{P_{m}(\cdot) ; m=1,2, \ldots\right\}$, их порождающая, определяет также случайный марковский ветвящийся процесс [10] $\left\{\mathfrak{k}_{m}=\left|\mathfrak{X}^{(m)}\right| ; m=0,1,2, \ldots\right\}$. Для этого процесса на основании формулы полной вероятности имеем

$$
\mathbf{P}\left\{\mathfrak{k}_{m+1}=i\right\}=\sum_{j=0}^{N^{m d}} \mathbf{P}\left\{\mathfrak{k}_{m+1}=i \mid \mathfrak{k}_{m}=j\right\} \mathbf{P}\left\{\mathfrak{k}_{m}=j\right\} .
$$

При этом условная вероятность $\mathbf{P}\left\{\mathfrak{k}_{m+1}=i \mid \mathfrak{k}_{m}=j\right\}$ вычисляется в терминах функции $q(\sigma)$. Используя $(8),(12)$, находим

$$
\begin{gathered}
\mathbf{P}\left\{\mathfrak{k}_{m+1}=i \mid \mathfrak{k}_{m}=j\right\}=\sum_{H: H \in \mathfrak{K}_{m+1},|H|=i} \mathbf{P}\left\{\mathbf{K}_{m+1}(\mathfrak{X})=H|| \mathbf{K}_{m}(\mathfrak{X}) \mid=j\right\}= \\
=\sum_{H: H \in \mathfrak{K}_{m+1},|H|=i} Q\left(H \mid \mathbf{K}_{m}(H)\right) \mathbf{P}\left\{\mathbf{K}_{m}(\mathfrak{X})=\mathbf{K}_{m}(H)|| \mathbf{K}_{m}(\mathfrak{X}) \mid=j\right\}= \\
=\sum_{H: H \in \mathfrak{K}_{m+1},|H|=i}\left(\prod_{\boldsymbol{\xi} \in \mathbf{K}_{m}(H)} q(\mathbf{T}(\boldsymbol{\xi} ; H))\right) \mathbf{P}\left\{\mathbf{K}_{m}(\mathfrak{X})=\mathbf{K}_{m}(H)|| \mathbf{K}_{m}(\mathfrak{X}) \mid=j\right\}
\end{gathered}
$$

или, применяя (6),

$$
\mathbf{P}\left\{\mathfrak{k}_{m+1}=i \mid \mathfrak{k}_{m}=j\right\}=\sum_{G: G \subset \mathfrak{K}_{m},|G|=j} Q_{i j}(G) \mathbf{P}\left\{\mathbf{K}_{m}(\mathfrak{X})=G|| \mathbf{K}_{m}(\mathfrak{X}) \mid=j\right\},
$$


где

$$
Q_{i j}(G)=\sum_{\left\{\sigma(\boldsymbol{\xi}): \boldsymbol{\xi} \in G, \mid \bigcup_{\boldsymbol{\xi} \in G}\right.} \prod_{(\boldsymbol{\xi}) \mid=i\}} q(\sigma(\boldsymbol{\xi})) .
$$

В формуле (14) мы использовали явный вид слагаемых, который допускает возможность рассматривать суммирование, входяшее в это выражение, как производяшееся по любым наборам $\{\sigma(\boldsymbol{\xi}) ; \boldsymbol{\xi} \in G\}$, для которых $\bigcup_{\boldsymbol{\xi} \in G}|\sigma(\boldsymbol{\xi})|=i$. Введем теперь обозначение

$$
\pi_{l}=\sum_{\sigma: \sigma \subset \Im_{N}^{d},|\sigma|=l} q(\sigma)
$$

и целочисленную характеристику $l(\boldsymbol{\xi})=|\sigma(\boldsymbol{\xi})|$ множеств, по набору которых производится суммирование в (14). Тогда в этих терминах вероятность $Q_{i j}(G)$ представима в виде

$$
\begin{aligned}
Q_{i j}(G) & =\sum_{\left\{l(\boldsymbol{\xi}): \boldsymbol{\xi} \in G, \sum_{\boldsymbol{\xi} \in G} l(\boldsymbol{\xi})=i\right\}} \sum_{\{\sigma(\boldsymbol{\xi}): \boldsymbol{\xi} \in G, l(\boldsymbol{\xi})=|\sigma(\boldsymbol{\xi})|\}} \prod_{\boldsymbol{\xi} \in G} q(\boldsymbol{\xi})= \\
& =\sum_{\left\{l(\boldsymbol{\xi}): \boldsymbol{\xi} \in G, \sum_{\boldsymbol{\xi} \in G} l(\boldsymbol{\xi})=i\right\}} \sum_{\boldsymbol{\xi} \in G} q(\boldsymbol{\xi})= \\
& =\sum_{\left\{l(\boldsymbol{\xi}): \boldsymbol{\xi} \in G, \sum_{\boldsymbol{\xi} \in G} l(\boldsymbol{\xi}) \subset \Im_{N}^{d},|\sigma(\boldsymbol{\xi})|=l(\boldsymbol{\xi})\right\}} \prod_{\boldsymbol{\xi} \in G} \pi_{l(\boldsymbol{\xi})} .
\end{aligned}
$$

В таком представлении вероятность $Q_{i j}(G) \equiv Q_{i j}$ при фиксированном $j=|G|$ не зависит от $G$. По этой причине из (13) следует

$$
\begin{aligned}
\mathbf{P}\left\{\mathfrak{k}_{m+1}=i \mid \mathfrak{k}_{m}=j\right\} & =Q_{i j} \sum_{G: G \subset \mathfrak{K}_{m},\left|\mathbf{K}_{m}(\mathfrak{X})\right|=j} \mathbf{P}\left\{\mathbf{K}_{m}(\mathfrak{X})=G|| G \mid=j\right\}= \\
& =Q_{i j} \mathbf{P}\left\{\left|\mathbf{K}_{m}(\mathfrak{X})\right|=j|| \mathbf{K}_{m}(\mathfrak{X}) \mid=j\right\}=Q_{i j} .
\end{aligned}
$$

Тогда при фиксированном $G$ с $|G|=j$ из соотношений (13), (15) следует, что условная вероятность перехода для процесса $\left\{\mathfrak{k}_{m} ; m=0,1,2, \ldots\right\}$ определяется выражением

$$
\mathbf{P}\left\{\mathfrak{k}_{m+1}=i \mid \mathfrak{k}_{m}=j\right\}=\sum_{\substack{l_{1}=1, \ldots, l_{j}=1, k \\ l_{1}+\cdots+l_{j}=i}}^{N^{d}} \prod_{k=1}^{j} \pi_{l_{k}} .
$$

Введем производящую функцию $\Psi_{m}(x), m=1,2, \ldots$, случайного числа $\mathfrak{k}_{m}$, характеризующую этот ветвящийся процесс [10],

$$
\Psi_{m}(x)=\sum_{l=1}^{N^{m d}} x^{l} \mathbf{P}\left\{\mathfrak{k}_{m}=l\right\}, \quad \Psi_{0}(x)=x .
$$

Эта функция удовлетворяет разностному уравнению,

$$
\Psi_{m+1}(x)=\Psi_{m}(\Pi(x)),
$$


где

$$
\Pi(x)=\sum_{l=1}^{N^{d}} x^{l} \pi_{l}, \quad \operatorname{deg} \Pi(x)=N^{d} .
$$

Уравнение получается на основе стандартного в теории ветвящихся процессов вычисления, используюшего выражения (16) и (11),

$$
\begin{aligned}
\Psi_{m+1}(x) & =\sum_{i=1}^{N^{(m+1) d}} x^{i} \sum_{j=1}^{N^{m d}} \mathbf{P}\left\{\mathfrak{k}_{m}=j\right\} \sum_{\substack{l_{1}=1, \ldots, l_{j}=1 \\
l_{1}+\cdots+l_{j}=i}}^{N^{d}} \prod_{k=1}^{j} \pi_{l_{k}}= \\
& =\sum_{j=1}^{N^{m d}} \mathbf{P}\left\{\mathfrak{k}_{m}=j\right\}(\Pi(x))^{j} .
\end{aligned}
$$

\section{7. ОСНОВНАЯ ТЕОРЕМА}

Самоусредняемость фрактальной размерности, определяемой для каждой случайной реализации $\mathfrak{X}$ формулой (2), влечет за собой возможность ее вычисления на основе математического ожидания $\left\langle\left|\mathfrak{X}^{(m)}\right|\right\rangle$, т.е. возможность заменить формулу (2) формулой

$$
D=\inf \left\{\alpha: \lim _{h \rightarrow 0} h^{\alpha}\left\langle\left|\mathfrak{X}^{(m)}\right|\right\rangle=0\right\} .
$$

Из уравнения (17) для производяшей функции дифференщированием по $x$ находим уравнение для математического ожидания $\left\langle\left|\mathfrak{X}^{(m+1)}\right|\right\rangle=a\left\langle\left|\mathfrak{X}^{(m)}\right|\right\rangle$, где $\left.a=\Pi^{\prime}(1)\right\rangle 1$. Тогда, учитьвая, что $\mathfrak{X}^{(0)}=\Lambda,\left|\mathbf{K}_{0}(\mathfrak{X})\right|=1$, получим $\left\langle\left|\mathbf{K}_{m}(\mathfrak{X})\right|\right\rangle=a^{m}$. Эта формула позволяет найти нижнюю грань в (18). Действительно, равенство

$$
\lim _{h \rightarrow 0} h^{\alpha}\left\langle\left|\mathfrak{X}^{(m)}\right|\right\rangle=\lim _{m \rightarrow \infty}\left(a N^{-\alpha}\right)^{m}=0
$$

имеет место в том и только в том случае, если $a<N^{D}$. Следовательно,

$$
D=\frac{\ln a}{\ln N}
$$

Заметим, что в точке $\alpha=D$ предел (18) конечен. Этот факт указывает на то, что допустимо сушествование предела $\lim _{h \rightarrow 0} h^{D}\left|\mathfrak{X}^{(m)}\right|$, который в этом случае является случайной величиной. Если такое положение имеет место, то на фрактальной структуре сушествует $D$-мера.

Докажем теперь высказанные предположения. Для начала вычислим двухточечные моменты случайного процесса $\left\{\mathfrak{k}_{m} ; m=0,1,2, \ldots\right\}$. Для этого нам понадобится двухточечная производяшая функция

$$
\Psi_{m, n}(x, y)=\sum_{i=1, j=1}^{\infty} x^{i} y^{j} \mathbf{P}\left\{\mathfrak{k}_{m}=i, \mathfrak{k}_{n}=j\right\}, \quad n>m
$$


Составим разностное уравнение по $n$ для значений $n>m$ этой функции при фиксированном $m$. Это уравнение получается буквальным повторением вычисления, проведенного в предыдушем разделе при выводе уравнения (17), т.к. вследствие свойства марковости случайного процесса $\left\{\mathfrak{k}_{m}\right\}$ при определении условных вероятностей исчезает условие $\mathfrak{k}_{m}=i$. В связи с этим получаем

$$
\Psi_{m, n+1}(x, y)=\Psi_{m, n}(x, \Pi(y))
$$

для всех значений $n \geqslant m$. Это разностное уравнение позволяет вычислять производяшую функцию $\Psi_{m, n}(x, y)$ в последовательные моменты "времени" $n=m+1, m+2, \ldots$ по известной функции $\Psi_{m, m}(x, y)$. Эта последняя функция с помошью определения (20) может быть записана в виде

$$
\Psi_{m, m}(x, y)=\sum_{i=0}^{\infty}(x y)^{i} \mathbf{P}\left\{\mathfrak{k}_{m}=i\right\}=\Psi_{m}(x y) .
$$

Беря данную функцию в качестве начального условия для уравнения (21), получим последовательными итерациями

$$
\Psi_{m, n}(x, y)=\Psi_{m}\left(x \Pi^{(n-m)}(y)\right),
$$

где $\Pi^{(n+1)}(y) \equiv \Pi\left(\Pi^{(n)}(y)\right), \Pi^{(0)}(x)=x$.

Вычислим теперь двухточечные моменты $\left\langle\mathfrak{k}_{m} \mathfrak{k}_{n}\right\rangle$ процесса $\left\{\mathfrak{k}_{m} ; m=0,1,2, \ldots\right\}$, которые при $n \neq m$ находятся согласно формуле

$$
\left\langle\mathfrak{k}_{m} \mathfrak{k}_{n}\right\rangle=\left.\frac{\partial^{2} \Psi_{m, n}(x, y)}{\partial x \partial y}\right|_{x=y=1} .
$$

Непосредственное вычисление дает нам связь между средними

$$
\left\langle\mathfrak{k}_{n} \mathfrak{k}_{m}\right\rangle=a^{n-m}\left\langle\mathfrak{k}_{m}^{2}\right\rangle .
$$

Из определения производяшей функции $\Psi_{m}(x)$ и уравнения (17) следует выражение для второго момента $\left\langle\mathfrak{k}_{m}^{2}\right\rangle=\Psi_{m}^{\prime \prime}(1)+\left\langle\mathfrak{k}_{m}\right\rangle$ в терминах величин $a$ и $a^{\prime}=\Pi^{\prime \prime}(1)$. В самом деле,

$$
\Psi_{m+1}^{\prime \prime}(1)=\Psi_{m}^{\prime}(1) a^{\prime}+\Psi_{m}^{\prime \prime}(1) a^{2}=a^{\prime} a^{m}+\Psi_{m}^{\prime \prime}(1) a^{2} .
$$

Используя решение этого разностного уравнения относительно $\Psi_{m}^{\prime \prime}(1)$ с начальным условием $\Psi_{m}^{\prime \prime}(1)=0$, находим

$$
\left\langle\mathfrak{k}_{m}^{2}\right\rangle=a^{\prime} a^{m-1} \frac{a^{m}-1}{a-1}+a^{m} .
$$

С целью доказательства основной теоремы введем случайньй процесс $\left\{\mathfrak{c}_{m} ; m=0,1\right.$, $2, \ldots\}, \mathfrak{c}_{m}=\mathfrak{k}_{m} / a^{m}$. Этот процесс обладает ковариацией $\mathbf{D}_{n, m}=\left\langle\left(\mathfrak{c}_{m}-\mathfrak{c}_{n}\right)^{2}\right\rangle$, определяемой формулой

$$
\mathbf{D}_{n, m}=\left(1+\frac{a^{\prime}}{a(a-1)}\right)\left(a^{-m}-a^{-n}\right), \quad n \geqslant m,
$$

что непосредственно следует из явных выражений (22), (23) для составляющих ее средних. Формула (24) позволяет доказать основной результат этой работы. 
Tеорема 2. Случайные множества класса $\mathbf{F}[q]$, у которых $\pi_{1}=0$, имеют неслучайную фрактальную размерность $D$, вычисляемую по формуле (19). На них с вероятностью 1 существует стохастическая D-мера.

ДокАЗАТЕльство. Покажем, что последовательность $\left\{\mathfrak{c}_{m} ; m=0,1,2, \ldots\right\}$ случайных величин сходится с вероятностью 1 к некоторой случайной величине с. Из формулы (24) следует на основании неравенства Чебышева оценка

$$
\mathbf{P}\left\{\left|\mathfrak{c}_{n}-\mathfrak{c}_{m}\right|>a^{-\frac{m}{4}}\right\}<a^{\frac{m}{2}} \mathbf{D}_{n, m}<\left(1+\frac{a^{\prime}}{a(a-1)}\right) a^{-\frac{m}{2}} .
$$

Так как $a>1$, то выражение в правой части суммируемо по $m$, т.е. последовательность $\mathfrak{c}_{n}$, рассматриваемая как последовательность функций на пространстве элементарных событий, сходится по вероятности, причем со скоростью геометрической прогрессии. Тогда применима теорема о сушествовании предела почти всюду на пространстве с мерой у последовательности измеримых функций $[11$, гл. V, $\S 4$, теорема 8]. Следовательно, последовательность $\left\{\mathfrak{c}_{m}\right\}$ действительно сходится с вероятностью 1. Это утверждение позволяет заключить, что с вероятностью 1 существует предел

$$
\lim _{m \rightarrow \infty} \frac{\mathfrak{k}_{m}}{a^{m}}=\lim _{m \rightarrow \infty} \frac{\left|\mathbf{K}_{m}(\mathfrak{X})\right|}{a^{m}}=\mathfrak{c} .
$$

Докажем, что с вероятностью единица $\mathfrak{c} \neq 0$. Введем функции $\Psi_{m}^{(l)}(x)$ и числовые последовательности $\left\{r_{l, m}\right\}$,

$$
\begin{gathered}
\Psi_{m}^{(l)}(x)=\int_{0}^{x} \Psi_{m}^{(l-1)}(y) d y, \quad \Psi_{m}^{(0)}(x)=\Psi_{m}(x) \\
r_{l, m}=a^{m} \int_{0}^{1} \Psi_{m}^{(l-1)}(x) d x=a^{m} \Psi_{m}^{(l)}(1), \quad m=1,2, \ldots, \quad l=1,2, \ldots
\end{gathered}
$$

Заметим, что $r_{l, m}=a^{m}\left\langle\left[\left(\mathfrak{k}_{m}+1\right)\left(\mathfrak{k}_{m}+2\right) \ldots\left(\mathfrak{k}_{m}+l\right)\right]^{-1}\right\rangle$. Получим для этих чисел рекуррентную оценку. На основании формулы Коши имеем

$$
\Psi_{m}^{(l)}(x)=[(l-1) !]^{-1} \int_{0}^{x}(x-y)^{l-1} \Psi_{m}(y) d y .
$$

Тогда, введя обратную функцию $\Pi^{(-1)}(\cdot)$, получим

$$
\begin{aligned}
\int_{0}^{1}(1-y)^{l-1} \Psi_{m+1}(y) d y & =a^{m+1} \int_{0}^{1}(1-y)^{l-1} \Psi_{m}(\Pi(y)) d y= \\
& =a^{m+1} \int_{0}^{1} \frac{\left(1-\Pi^{(-1)}(y)\right)^{l-1}}{\Pi^{\prime}\left(\Pi^{(-1)}(y)\right)} \Psi_{m}(y) d y
\end{aligned}
$$

и положим $r_{l}(y)=-l^{-1}\left[\left(1-\Pi^{(-1)}(y)\right)^{l}\right]^{\prime}$. Используя функцию $\Psi_{m}(y)$, построим оценку для чисел

$$
r_{l, m+1}=a^{m+1}[(l-1) !]^{-1} \int_{0}^{1} r_{l}(y) \Psi_{m}(y) d y .
$$


Интегрируя $(l-1)$ раз по частям в последнем равенстве и считая, что $r_{l}^{(s)}(1)=0, s<$ $l-1$, получаем

$$
\begin{aligned}
r_{l, m+1} & =a^{m+1}[(l-1) !]^{-1} \int_{0}^{1} r_{l}^{(l-1)}(y) \Psi_{m}^{(l-1)}(y) d y= \\
& =(-1)^{l-1} a^{m+1}[(l-1) !]^{-1}\left(r_{l}^{(l-1)}(1) \Psi_{m}^{(l)}(1)-\int_{0}^{1} r_{l}^{(l)}(y) \Psi_{m}^{(l)}(y) d y\right) .
\end{aligned}
$$

Так как $r^{(l-1)}(1)=(-1)^{l-1}(l-1) ! a^{-l}$ при $\Pi^{\prime}(1)=a, \Pi(1)=1$, то искомая оценка имеет вид $r_{l, m+1} \leqslant a^{1-l} r_{l, m}+C_{l} r_{l+1, m}$, где

$$
C_{l}=a[(l-1) !]^{-1}\left(r_{l}^{(l)}(1)+\int_{0}^{1}\left|r_{l}^{(l+1)}(x)\right| d x\right) .
$$

Отсюда итерациями по $m$ находим неравенство

$$
r_{k, m}<a^{(1-k) m}\left(r_{k, 0}+C_{k} \sum_{n=0}^{\infty} r_{k+1, n}\right),
$$

справедливое для $k=1,2, \ldots, l$. В случае сходимости ряда в правой части (25) для некоторого $k$ из этой оценки следует сходимость такого же ряда для $k-1$, если $k>1$.

При выполнении условия $\pi_{1}=0$ с вероятностью 1 выполняется неравенство $\mathfrak{k}_{m} \geqslant$ $2^{m}$. Поэтому, взяв число $0<\sigma<1$, можно выбрать такое $l$, что $a^{m \mathfrak{k}_{m}^{-(l+1)}}<\sigma^{m}$. Следовательно, с вероятностью 1 сходится ряд

$$
\sum_{m=0}^{\infty} a^{m}\left\langle\mathfrak{k}_{m}^{-(l+1)}\right\rangle \text {. }
$$

Тогда, осушествляя спуск по $k=l, l-1, \ldots, 2$, получим, что сходится ряд

$$
\sum_{m=0}^{\infty} r_{2, m}
$$

Поэтому из неравенства (25) при $k=1$ следует, что с вероятностью 1 имеет место неравенство

$$
r_{1, m}<r_{1,0}+C_{1} \sum_{n=0}^{\infty} r_{2, n} .
$$

Следовательно, сушествует точная верхняя грань

$$
\sup _{m} a^{m}\left\langle\mathfrak{k}_{m}^{-1}\right\rangle=\sup _{m} r_{1, m} \leqslant r_{1,0}+C_{1} \sum_{n=0}^{\infty} r_{2, n}<\infty .
$$

Тогда $\left\langle\mathfrak{c}^{-1}\right\rangle=\lim _{m \rightarrow \infty}\left\langle\mathfrak{c}_{m}^{-1}\right\rangle \leqslant \sup _{m} a^{m}\left\langle\mathfrak{k}_{m}^{-1}\right\rangle<\infty$. Поэтому $\mathfrak{c}^{-1}<\infty$ с вероятностью 1. 
Из существования ненулевого предела с следует, что, во-первых, нижняя грань

$$
D=\inf \left\{\alpha: \lim _{m \rightarrow \infty} N^{-\alpha m}\left|\mathbf{K}_{m}(\mathfrak{X})\right|=0\right\}
$$

определяется из уравнения $N^{D}=a$ и поэтому она не является случайной, а, во-вторых, выбрав это значение $D$, мы получаем, что с вероятностью 1 существует предел

$$
\lim _{m \rightarrow \infty} N^{-m D}\left|\mathbf{K}_{m}(\mathfrak{X})\right|=\mathfrak{c} \neq 0 .
$$

Это означает существование с вероятностью 1 стохастической $D$-меры на реализациях случайного множества. Теорема 2 доказана.

\section{8. ЗАКЛЮЧЕНИЕ}

Построение стохастической геометрии для описания неупорядоченных сред есть необходимый шаг, предваряющий построение статистической механики физических полей на них. В данной работе дан конструктивньй метод вероятностного описания фрактально-неупорядоченных сред. Точечные случайные поля, появляюшиеся при этом, не являются ни ординарными, ни сепарабельными. В рамках предложенной схемы введен обширный класс точечных случайных полей, которые названы нами полями с марковскими измельчениями. Для этого класса полей доказана теорема о самоусредняемости их фрактальной размерности $D$. При этом фрактальная мера, определяемая на таких геометрических структурах, не является стохастической, а есть $D$-мера. Это позволяет ввести на фрактале стохастический интеграл и на его основе феноменологический функционал свободной энергии, т.е. построить на нем статистическую механику.

\section{Список литературы}

[1] J. Zaiman. Models of Disorder. The Theoretical Physics of Homogeneously Disordered Systems. Cambridge: Cambridge University Press, 1979.

[2] И. И. Гихман, А. В. Скороход. Введение в теорию случайных процессов. М.: Наука, 1977.

[3] В. З. Беленький. Геометрико-вероятностные модели кристаллизации. М.: Наука, 1980.

[4] K. Matthes, J. Kerstan, J. Mecke. Infinitely Divisible Point Processes. Berlin: Academie-Verlag; New York: John Wiley \& Sons 1978.

[5] Г. Федерер. Геометрическая теория меры. М.: Наука, 1987.

[6] P. R. Massopust. Fractal Functions, Fractal Surfaces, and Wavelets. New York: Academic Press, 1994.

[7] M. F. Barnsley. Fractals Everywhere. Orlando, Florida: Academic Press, 1988.

[8] К. . Партасарати. Введение в теорию вероятностей и теорию меры. М.: Мир, 1983.

[9] A. Ya. Virchenko, A. Ya. Dulfan. Functional Materials. 1998. V. 5. № 4. P. 471-474.

[10] Б. А. Севастьянов. Ветвящиеся процессы. М.: Наука, 1971.

[11] А. Н. Колмогоров, С. В. Фомин. Элементы теории функций и функционального анализа. М.: Наука, 1972. 